International Journal of Pure and Applied Mathematics

Volume 107 No. 1 2016, 279-288

ISSN: 1311-8080 (printed version); ISSN: 1314-3395 (on-line version)

url: http://www.ijpam.eu

doi: 10.12732/ijpam.v107i1.20

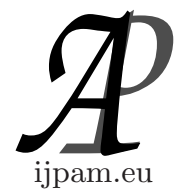

\title{
SOFT $L$-FUZZY QUASI-UNIFORMITIES INDUCED BY SOFT $L$-NEIGHBORHOOD SYSTEMS
}

\author{
Jung Mi Ko${ }^{1}$, Yong Chan $\mathrm{Kim}^{2} \S$ \\ ${ }^{1,2}$ Department of Mathematics \\ Gangneung-Wonju University \\ Gangneung, Gangwondo, 210-702, KOREA
}

\begin{abstract}
In this paper, we obtain soft $L$-fuzzy quasi-uniformities induced by soft $L$ neighborhood systems in complete residuated lattices. Moreover, every $N$-continuous surjective soft maps are uniformly continuous soft maps. We give their examples.
\end{abstract}

AMS Subject Classification: 03E72, 06A15, 06F07, 54A05

Key Words: complete residuated lattice, soft $L$-neighborhood space, soft $L$-fuzzy quasiuniform space, $N$-continuous soft maps, uniformly continuous soft maps

\section{Introduction}

Molodtsov [13] introduced the soft set as a mathematical tool for dealing information as the uncertainty of data in engineering, physics, computer sciences and many other diverse field. Presently, the soft set theory is making progress rapidly $[1,3,4,9.10,16,17,19,20]$. Pawlak's rough set $[14,15]$ can be viewed as a special case of soft rough sets [4].

Kim $[9,10]$ introduced a fuzzy soft $F: A \rightarrow L^{U}$ as an extension as the soft $F: A \rightarrow P(U)$ where $L$ is a complete residuated lattice $[2,5,6]$. He introduced soft $L$-fuzzy interior and closure operators, quasi-uniformities and soft $L$-fuzzy topogenous orders in complete residuated lattices.

In this paper, we obtain soft $L$-fuzzy quasi-uniformities induced by soft $L$-neighborhood systems in complete residuated lattices. Moreover, every $\mathrm{N}$ continuous surjective soft maps are uniformly continuous soft maps. We give

Received: November 11, 2016

Published: March 31, 2016

${ }^{\S}$ Correspondence author (c) 2016 Academic Publications, Ltd.

url: www.acadpubl.eu 
their examples.

\section{Preliminaries}

Definition 2.1. $[2,5,6]$ An algebra $(L, \wedge, \vee, \odot, \rightarrow, 0,1)$ is called a complete residuated lattice if it satisfies the following conditions:

(C1) $L=(L, \leq, \vee, \wedge, 1,0)$ is a complete lattice with the greatest element 1 and the least element 0 ;

(C2) $(L, \odot, 1)$ is a commutative monoid;

(C3) $x \odot y \leq z$ iff $x \leq y \rightarrow z$ for $x, y, z \in L$.

In this paper, we assume that $(L, \leq, \odot, \rightarrow)$ is a complete residuated lattice and we denote $L_{0}=L-\{0\}$.

Lemma 2.2. $[2,5,6]$ For each $x, y, z, x_{i}, y_{i}, w \in L$, we have the following properties.

(1) $1 \rightarrow x=x, 0 \odot x=0$,

(2) If $y \leq z$, then $x \odot y \leq x \odot z, x \rightarrow y \leq x \rightarrow z$ and $z \rightarrow x \leq y \rightarrow x$,

(3) $x \odot y \leq x \wedge y \leq x \vee y \leq x \oplus y$,

(4) $x \odot\left(\bigvee_{i} y_{i}\right)=\bigvee_{i}\left(x \odot y_{i}\right)$,

(5) $x \rightarrow\left(\bigwedge_{i} y_{i}\right)=\bigwedge_{i}\left(x \rightarrow y_{i}\right)$,

(6) $\left(\bigvee_{i} x_{i}\right) \rightarrow y=\bigwedge_{i}\left(x_{i} \rightarrow y\right)$,

(7) $x \rightarrow\left(\bigvee_{i} y_{i}\right) \geq \bigvee_{i}\left(x \rightarrow y_{i}\right)$,

(8) $\left(\bigwedge_{i} x_{i}\right) \rightarrow y \geq \bigvee_{i}\left(x_{i} \rightarrow y\right)$,

(9) $(x \odot y) \rightarrow z=x \rightarrow(y \rightarrow z)=y \rightarrow(x \rightarrow z)$,

(10) $x \odot(x \rightarrow y) \leq y$ and $x \rightarrow y \leq(y \rightarrow z) \rightarrow(x \rightarrow z)$,

(11) $(x \rightarrow y) \odot(z \rightarrow w) \leq(x \odot z) \rightarrow(y \odot w)$,

(12) $x \rightarrow y \leq(x \odot z) \rightarrow(y \odot z)$ and $(x \rightarrow y) \odot(y \rightarrow z) \leq x \rightarrow z$.

Definition 2.3. $[9,10]$ Let $X$ be an initial universe of objects and $E$ the set of parameters (attributes) in $X$. A pair $(F, A)$ is called a fuzzy soft set over $X$, where $A \subset E$ and $F: A \rightarrow L^{X}$ is a mapping. We denote $S(X, A)$ as the family of all fuzzy soft sets under the parameter $A$.

Definition 2.4. $[9,10]$ Let $(F, A)$ and $(G, A)$ be two fuzzy soft sets over a common universe $X$.

(1) $(F, A)$ is a fuzzy soft subset of $(G, A)$, denoted by $(F, A) \leq(G, A)$ if $F(a) \leq G(a)$, for each $a \in A$. 
(2) $(F, A) \wedge(G, A)=(F \wedge G, A)$ if $(F \wedge G)(a)=F(a) \wedge G(a)$ for each $a \in A$.

(3) $(F, A) \vee(G, A)=(F \vee G, A)$ if $(F \vee G)(a)=F(a) \vee G(a)$ for each $a \in A$.

(4) $(F, A) \odot(G, A)=(F \odot G, A)$ if $(F \odot G)(a)=F(a) \odot G(a)$ for each $a \in A$.

(6) $\alpha \odot(F, A)=(\alpha \odot F, A)$ for each $\alpha \in L$.

Definition 2.5. $[9,10]$ Let $S(X, A)$ and $S(Y, B)$ be the families of all fuzzy soft sets over $X$ and $Y$, respectively. The mapping $f_{\phi}: S(X, A) \rightarrow S(Y, B)$ is a soft mapping where $f: X \rightarrow Y$ and $\phi: A \rightarrow B$ are mappings.

(1) The image of $(F, A) \in S(X, A)$ under the mapping $f_{\phi}$ is denoted by $f_{\phi}((F, A))=\left(f_{\phi}(F), B\right)$ where

$$
\begin{aligned}
& f_{\phi}(F)(b)(y)= \\
& \begin{cases}\bigvee_{a \in \phi^{-1}(\{b\})} f^{\rightarrow}(F(a))(y), & \text { if } \phi^{-1}(\{b\}) \neq \emptyset, \\
0, & \text { otherwise. }\end{cases}
\end{aligned}
$$

(2) The inverse image of $(G, B) \in S(Y, B)$ under the mapping $f_{\phi}$ is denoted by $f_{\phi}^{-1}((G, B))=\left(f_{\phi}^{-1}(G), A\right)$ where

$$
f_{\phi}^{-1}(G)(a)(x)=f^{\leftarrow}(G(\phi(a)))(x), \forall a \in A, x \in X .
$$

(3) The soft mapping $f_{\phi}: S(X, A) \rightarrow S(Y, B)$ is called injective (resp. surjective, bijective) if $f$ and $\phi$ are both injective (resp. surjective, bijective).

Lemma 2.6. $[9,10]$ Let $f_{\phi}: S(X, A) \rightarrow S(Y, B)$ be a soft mapping. Then we have the following properties. For $(F, A),\left(F_{i}, A\right) \in S(X, A)$ and $(G, B),\left(G_{i}, B\right) \in S(Y, B)$,

(1) $(G, B) \geq f_{\phi}\left(f_{\phi}^{-1}((G, B))\right)$ with equality if $f$ is surjective,

(2) $(F, A) \leq f_{\phi}^{-1}\left(f_{\phi}((F, A))\right)$ with equality if $f$ is injective,

(3) $f_{\phi}^{-1}\left(\bigvee_{i \in I}\left(G_{i}, B\right)\right)=\bigvee_{i \in I} f_{\phi}^{-1}\left(\left(G_{i}, B\right)\right)$,

(4) $f_{\phi}^{-1}\left(\bigwedge_{i \in I}\left(G_{i}, B\right)\right)=\bigwedge_{i \in I} f_{\phi}^{-1}\left(\left(G_{i}, B\right)\right)$,

(5) $f_{\phi}\left(\bigvee_{i \in I}\left(F_{i}, A\right)\right)=\bigvee_{i \in I} f_{\phi}\left(\left(F_{i}, A\right)\right)$

(6) $f_{\phi}\left(\bigwedge_{i \in I}\left(F_{i}, A\right)\right) \leq \bigwedge_{i \in I} f_{\phi}\left(\left(F_{i}, A\right)\right)$ with equality if $f$ is injective,

(7) $f_{\phi}^{-1}\left(\left(G_{1}, B\right) \odot\left(G_{2}, B\right)\right)=f_{\phi}^{-1}\left(\left(G_{1}, B\right)\right) \odot f_{\phi}^{-1}\left(\left(G_{2}, B\right)\right)$,

(8) $f_{\phi}\left(\left(F_{1}, A\right) \odot\left(F_{2}, A\right)\right) \leq f_{\phi}\left(\left(F_{1}, A\right)\right) \odot f_{\phi}\left(\left(F_{2}, A\right)\right)$ with equality if $f$ is injective.

Definition 2.7. [9] A map $N: X \rightarrow\left(L^{A}\right)^{S(X, A)}$ is called a soft $L$ neighborhood system on $X$ if $N=\left\{N_{x}=N(x) \mid x \in X\right\}$ satisfies the following conditions

$(\mathrm{SN} 1) N_{x}\left(\left(1_{X}, A\right)\right)=\left(1_{X}, A\right)(x)=1_{A}$ and $N_{x}\left(\left(1_{X}, A\right)\right)=\left(0_{X}, A\right)(x)=0_{A}$, 
$(\mathrm{SN} 2) N_{x}((F, A) \odot(G, A)) \geq N_{x}((F, A)) \odot N_{x}((G, A))$ for each $(F, A),(G, A) \in$ $S(X, A)$,

(SN3) If $(F, A) \leq(G, A)$, then $N_{x}((F, A)) \leq N_{x}((G, A))$,

(SN4) $N_{x}((F, A)) \leq(F, A)(x)$ for all $(F, A) \in S(X, A)$ where $(F, A)(x)=$ $F(-)(x)$.

A soft $L$-neighborhood system is called stratified if

(S) $N_{x}(\alpha \odot(F, A)) \geq \alpha \odot N_{x}((F, A))$ for all $(F, A) \in S(X, A)$ and $\alpha \in L$.

The triple $(X, A, N)$ is called a soft $L$-neighborhood space.

Let $(X, A, N)$ and $(Y, B, M)$ be soft $L$-neighborhood spaces. A mapping $f_{\phi}:(X, A, N) \rightarrow(Y, B, M)$ is said to be an $N$-continuous soft map iff

$$
M_{f(x)}((G, B))(\phi(a)) \leq N_{x}\left(f_{\phi}^{-1}((G, B))\right)(a)
$$

for each $x \in X, a \in A,(G, B) \in S(Y, B)$.

Definition 2.8. [10] A mapping $\mathcal{U}: S(X \times X, A) \rightarrow L$ is called a soft $L$-fuzzy quasi-uniformity on $X$ iff it satisfies the properties.

(SU1) There exists $(U, A) \in S(X \times X, A)$ such that $\mathcal{U}((U, A))=1$,

(SU2) If $(V, A) \leq(U, A)$, then $\mathcal{U}((V, A)) \leq \mathcal{U}((U, A))$,

(SU3) For every $(U, A),(V, A) \in S(X \times X, A)$,

$$
\mathcal{U}((U, A) \odot(V, A)) \geq \mathcal{U}((U, A)) \odot \mathcal{U}((V, A))
$$

(SU4) If $\mathcal{U}((U, A)) \neq 0$, then $\left(1_{\triangle}, A\right) \leq(U, A)$, where

$$
1_{\Delta}(a)(x, y)= \begin{cases}1 & \text { if } x=y \\ 0 & \text { if } x \neq y\end{cases}
$$

$(\mathrm{SU} 5) \bigvee\{\mathcal{U}((V, A)) \mid(V, A) \circ(V, A) \leq(U, A)\} \geq \mathcal{U}((U, A))$

$$
\begin{aligned}
& ((V, A) \circ(V, A))(a)(x, y)=(V(a) \circ V(a))(x, y) \\
& =\bigvee_{z \in X}(V(a)(z, x) \odot V(a)(x, y)), \quad \forall x, y \in X, a \in A
\end{aligned}
$$

The triple $(X, A, \mathcal{U})$ is called a soft $L$-fuzzy quasi-uniform space.

Let $\left(X, A, \mathcal{U}_{X}\right)$ and $\left(Y, B, \mathcal{U}_{Y}\right)$ be soft $L$-fuzzy quasi-uniform spaces and $f_{\phi}:(X, A) \rightarrow(Y, B)$ be a soft map. Then $f_{\phi}:\left(X, A, \mathcal{U}_{X}\right) \rightarrow\left(Y, B, \mathcal{U}_{Y}\right)$ is called an uniformly continuous soft map if for all $(V, B) \in S(Y \times Y, B)$,

$$
\mathcal{U}_{Y}((V, B)) \leq \mathcal{U}_{X}\left((f \times f)_{\phi}^{-1}((V, B))\right), \forall(V, B) \in S(Y \times Y, B) .
$$




\section{Soft $L$-Fuzzy Quasi-Uniformities Induced by Soft $L$-Neighborhood Systems}

Lemma 3.1. For every $(F, A),(G, A) \in S(X, A)$, we define $\left(U_{F}, A\right),\left(U_{F}^{-1}, A\right) \in$ $S(X \times X, A)$ by

$$
U_{F}(a)(x, y)=F(a)(x) \rightarrow F(a)(y)
$$

Then we have the following properties.

(1) $\left(1_{X \times X}, A\right)=\left(U_{0_{X}}, A\right)=\left(U_{1_{X}}, A\right)$,

(2) $\left(1_{\triangle}, A\right) \leq\left(U_{F}, A\right)$

(3) $\left(U_{F}, A\right) \circ\left(U_{F}, A\right)=\left(U_{F}, A\right)$,

(4) $\left(U_{F}, A\right) \odot\left(U_{G}, A\right) \leq\left(U_{F \odot G}, A\right)$.

Proof. (1)

$$
\begin{aligned}
& 1_{X \times X}(a)(x, y)=1=U_{0_{X}}(a)(x, y) \\
& =0_{X}(a)(x) \rightarrow 0_{X}(a)(y) \\
& =1_{X}(a)(x) \rightarrow 1_{X}(a)(y)=U_{1_{X}}(a)(x, y) .
\end{aligned}
$$

(2) Since $U_{F}(a)(x, x)=F(a)(x) \rightarrow F(a)(x)=0,\left(1_{\triangle}, A\right) \leq\left(U_{F}, A\right)$.

(3) $\left(U_{F}, A\right) \circ\left(U_{F}, A\right) \leq\left(U_{F}, A\right)$ from

$$
\begin{aligned}
& \left(U_{F}(a) \circ U_{F}(a)\right)(x, z) \\
& =\bigvee_{y \in X}\left(U_{F}(a)(x, y) \circ U_{F}(a)(y, z)\right) \\
& =\bigvee_{y \in X}((F(a)(x) \rightarrow F(a)(y)) \odot(F(a)(y) \rightarrow F(a)(z))) \\
& \leq F(a)(x) \rightarrow F(a)(z)=U_{F}(a)(x, z) .
\end{aligned}
$$

$\left(U_{F}, A\right) \circ\left(U_{F}, A\right) \geq\left(U_{F}, A\right)$ from

$$
\begin{aligned}
& \left(U_{F}(a) \circ U_{F}(a)\right)(x, z) \\
& =\bigvee_{y \in X}\left(U_{F}(a)(x, y) \circ U_{F}(a)(y, z)\right) \\
& \geq((F(a)(x) \rightarrow F(a)(x)) \odot(F(a)(x) \rightarrow F(a)(z))) \\
& =U_{F}(a)(x, z)
\end{aligned}
$$

(4) By Lemma $2.2(11)$,

$$
\begin{aligned}
& U_{F}(a)(x, y) \odot U_{G}(a)(x, y) \\
& =(F(a)(x) \rightarrow F(a)(y)) \odot(G(a)(x) \rightarrow G(a)(y)) \\
& \leq(F(a)(x) \odot G(a)(x) \rightarrow F(a)(y) \odot G(a)(y) \\
& =U_{F \odot G}(a)(x, y) .
\end{aligned}
$$


Theorem 3.2. Let $(X, A, N)$ be a soft $L$-neighborhood space. Define a $\operatorname{map} \mathcal{U}_{N}: S(X \times X) \rightarrow L$ by

$$
\mathcal{U}_{N}((U, A))=\bigvee\left\{\bigwedge_{a \in A} \bigvee_{x \in X} \odot_{i=1}^{n} N_{x}\left(\left(F_{i}, A\right)\right)(a) \mid \odot_{i=1}^{n}\left(U_{F_{i}}, A\right) \leq(U, A)\right\} .
$$

Then

(1) $\left(X, A, \mathcal{U}_{N}\right)$ is a soft $L$-fuzzy quasi-uniform space.

(2) If $N$ is stratified, then $\mathcal{U}_{N}$ is stratified.

Proof. (SU1) Since $\left(U_{1_{X}}, A\right)=\left(1_{X \times X}, A\right)$, we have

$$
\mathcal{U}_{N}\left(\left(1_{X \times X}, A\right)\right) \geq \bigvee_{x \in X, a \in A} N_{x}\left(\left(1_{X}, A\right)\right)(a)=1
$$

(SU2) If $\left(U_{1}, A\right) \leq\left(U_{2}, A\right),\left(U_{1}, A\right),\left(U_{2}, A\right) \in S(X \times X, A)$, then

$$
\begin{aligned}
& \mathcal{U}_{N}\left(\left(U_{1}, A\right)\right)=\bigvee\left\{\bigvee_{x \in X, a \in A} N_{x}((F, A)) \mid\left(U_{F}, A\right) \leq\left(U_{1}, A\right)\right\} \\
& \leq \bigvee\left\{\bigvee_{x \in X, a \in A} N_{x}((F, A)) \mid\left(U_{F}, A\right) \leq\left(U_{2}, A\right)\right\}=\mathcal{U}_{N}\left(\left(U_{2}, A\right)\right)
\end{aligned}
$$

(SU3)

$$
\begin{aligned}
& \mathcal{U}_{N}((U, A)) \odot \mathcal{U}_{N}((V, A)) \\
& \bigvee\left\{\bigwedge_{a \in A} \bigvee_{x \in X} \odot_{i=1}^{n} N_{x}\left(\left(F_{i}, A\right)\right)(a) \mid \odot_{i=1}^{n}\left(U_{F_{i}}, A\right) \leq(U, A)\right\} \\
& \left.\odot \bigvee \bigwedge_{a \in A} \bigvee_{x \in X} \odot_{i=1}^{n} N_{x}\left(\left(G_{j}, A\right)\right)(a) \mid \odot_{j=1}^{m}\left(U_{G_{j}}, A\right) \leq(V, A)\right\} \\
& \leq \bigvee\left\{\bigwedge _ { a \in A } \bigvee _ { x \in X } N _ { x } \left(\odot _ { i = 1 } ^ { n } N _ { x } ( ( F _ { i } , A ) ) ( a ) \odot \left(\odot_{i=1}^{n} N_{x}\left(\left(F_{i}, A\right)\right)(a)\right.\right.\right. \\
& \left.\mid \odot_{i=1}^{n}\left(U_{F_{i}}, A\right) \odot \odot_{i=1}^{n}\left(U_{F_{i}}, A\right) \leq(U, A) \odot(V, A)\right\} \\
& \leq \mathcal{U}_{N}((U, A) \odot(V, A)) .
\end{aligned}
$$

(SU4) If $\mathcal{U}_{N}((U, A)) \neq \perp$, then there exist $\left(F_{i}, A\right) \in S(X, A)$ with $\odot_{i=1}^{n}\left(U_{F_{i}}, A\right)$ $\leq(U, A)$ such that

$$
\bigwedge_{a \in A} \bigvee_{x \in X} \odot_{i=1}^{n} N_{x}\left(\left(F_{i}, A\right)\right)(a) \neq 0
$$

For all $a \in A$ and for some $x \in X$,

$$
\odot_{i=1}^{n} N_{x}\left(\left(F_{i}, A\right)\right)(a) \neq 0 .
$$

By Lemma 3.1, $1_{\triangle}(a) \leq U_{F_{i}}(a)$, Hence $\left(1_{\triangle}, A\right) \leq \odot_{i=1}^{n}\left(U_{F_{i}}, A\right) \leq(U, A)$.

(SU5) Suppose there exists $(U, A) \in S(X \times X, A)$ such that

$$
\bigvee\left\{\mathcal{U}_{N}((V, A)) \mid(V, A) \circ(V, A) \leq(U, A)\right\} \nsupseteq \mathcal{U}_{N}((U, A)) .
$$


Then there exist $\left(F_{i}, A\right) \in S(X, A)$ with $\odot_{i=1}^{n}\left(U_{F_{i}}, A\right) \leq(U, A)$ such that

$$
\bigvee\left\{\mathcal{U}_{N}((V, A)) \mid(V, A) \circ(V, A) \leq(U, A)\right\} \nsupseteq \bigwedge_{a \in A} \bigvee_{x \in X} \odot_{i=1}^{n} N_{x}\left(\left(F_{i}, A\right)\right)(a) .
$$

$\operatorname{Put}(W, A)=\odot_{i=1}^{n}\left(U_{F_{i}}, A\right)$. Then

$$
\begin{aligned}
& (W, A) \circ(W, A)=\left(\odot_{i=1}^{n}\left(U_{F_{i}}, A\right)\right) \circ\left(\odot_{i=1}^{n}\left(U_{F_{i}}, A\right)\right) \\
& =\odot_{i=1}^{n}\left(\left(U_{F_{i}}, A\right) \circ\left(U_{F_{i}}, A\right)\right)=\odot_{i=1}^{n}\left(U_{F_{i}}, A\right) \leq(U, A) . \\
& \quad \bigvee\left\{\mathcal{U}_{N}((V, A)) \mid(V, A) \circ(V, A) \leq(U, A)\right\} \\
& \quad \geq \mathcal{U}_{N}((W, A)) \geq \bigwedge_{a \in A} \bigvee_{x \in X} \odot_{i=1}^{n} N_{x}\left(\left(F_{i}, A\right)\right)(a) .
\end{aligned}
$$

It is a contradiction.

(2) Let $(U, A) \in S(X \times X, A),(F, A) \in S(X, A)$ and $\alpha \in L$, we have

$$
\begin{aligned}
& \mathcal{U}_{N}(\alpha \odot(U, A)) \\
& =\bigvee\left\{\bigwedge_{a \in A} \bigvee_{x \in X} \odot_{i=1}^{n} N_{x}\left(\left(F_{i}, A\right)\right)(a) \mid \odot_{i=1}^{n}\left(U_{F_{i}}, A\right) \leq \alpha \odot(U, A)\right\} \\
& \geq \bigvee\left\{\bigwedge_{a \in A} \bigvee_{x \in X} \odot_{i=1}^{n} N_{x}\left(\left(\alpha \odot F_{i}, A\right)\right)(a) \mid \odot_{i=1}^{n}\left(U_{\alpha \odot F_{i}}, A\right) \leq \alpha \odot(U, A)\right\} \\
& \geq \bigvee\left\{\alpha \odot \bigwedge_{a \in A} \bigvee_{x \in X} \odot_{i=1}^{n} N_{x}\left(\left(F_{i}, A\right)\right)(a) \mid \odot_{i=1}^{n}\left(U \alpha \odot F_{i}, A\right) \leq \alpha \odot(U, A)\right\} \\
& \geq \bigvee\left\{\alpha \odot \bigwedge_{a \in A} \bigvee_{x \in X} \odot_{i=1}^{n} N_{x}\left(\left(F_{i}, A\right)\right)(a) \mid \odot_{i=1}^{n}\left(U_{F_{i}}, A\right) \leq(U, A)\right\} \\
& \geq \alpha \odot \mathcal{U}_{N}((U, A)) .
\end{aligned}
$$

Theorem 3.3. Let $(X, A, N)$ and $(Y, B, M)$ be soft $L$-neighborhood spaces. Let $f_{\phi}:(X, A, N) \rightarrow(Y, B, M)$ be $N$-continuous surjective soft map. Then $f_{\phi}:\left(X, A, \mathcal{U}_{N}\right) \rightarrow\left(Y, B, \mathcal{V}_{M}\right)$ is an uniformly continuous soft map.

Proof.

$$
\begin{aligned}
& (f \times f)_{\phi}^{-1}\left(U_{G}\right)(a)\left(x_{1}, x_{2}\right)=U_{G}(\phi(a))\left(f\left(x_{1}\right), f\left(x_{2}\right)\right) \\
& =G(\phi(a))\left(f\left(x_{1}\right)\right) \rightarrow G(\phi(a))\left(f\left(x_{2}\right)\right) \\
& =f_{\phi}^{-1}(G)(a)\left(x_{1}\right) \rightarrow f_{\phi}^{-1}(G)(a)\left(x_{2}\right) \\
& =U_{f_{\phi}^{-1}(G)}(a)\left(x_{1}, x_{2}\right)
\end{aligned}
$$

Thus,

$$
\begin{aligned}
& \mathcal{V}_{M}((V, A)) \\
& =\bigvee\left\{\bigwedge_{b \in B} \bigvee_{y \in Y} \odot_{i=1}^{n} M_{y}\left(\left(G_{i}, B\right)\right)(b) \mid \odot_{i=1}^{n}\left(U_{G_{i}}, A\right) \leq(V, B)\right\} \\
& \left(f_{\phi} \text { is surjective }\right) \\
& =\bigvee\left\{\bigwedge_{a \in A} \bigvee_{x \in X} \odot_{i=1}^{n} M_{f(x)}\left(\left(G_{i}, B\right)\right)(\phi(a)) \mid\right. \\
& \left.\odot_{i=1}^{n}(f \times f)_{\phi}^{-1}\left(\left(U_{G_{i}}, A\right)\right) \leq(f \times f)_{\phi}^{-1}((V, B))\right\} \\
& \left(f_{\phi} \text { is } N \text {-continuous }\right) \\
& =\bigvee\left\{\bigwedge_{a \in A} \bigvee_{x \in X} \odot_{i=1}^{n} N_{x}\left(f_{\phi}^{-1}\left(G_{i}, B\right)\right)(a) \mid\right. \\
& \left.\left.\odot_{i=1}^{n}\left(U_{f_{\phi}^{-1}\left(G_{i}\right)}, A\right)\right) \leq(f \times f)_{\phi}^{-1}((V, B))\right\} \\
& \left.\leq \mathcal{U}_{N}\left((f \times f)_{\phi}^{-1}(V, B)\right)\right) .
\end{aligned}
$$


Example 3.4. Let $U=\left\{h_{i} \mid i=\{1, \ldots, 6\}\right\}$ with $h_{i}=$ house and $E=$ $\{e, b, w, c, i\}$ with $e=$ expensive, $b=$ beautiful, $w=$ wooden, $c=$ creative, $i=$ in the green surroundings.

Define a binary operation $\odot$ on $[0,1]$ by

$$
\begin{gathered}
x \odot y=\max \{0, x+y-1\}, x \rightarrow y=\min \{1-x+y, 1\} \\
x \oplus y=\min \{1, x+y\}, x^{*}=1-x
\end{gathered}
$$

Then $([0,1], \wedge, \rightarrow, 0,1)$ is a complete residuated lattice (ref.[2,7,21]). Let $A=$ $\{b, c\} \subset E$ and $X=\left\{h_{1}, h_{4}, h_{5}\right\}$. Put $(H, A)$ be a fuzzy soft set as follow:

$$
\begin{array}{ccccc}
(H, A) & h_{1} & h_{4} & h_{5} & \\
b & 0.5 & 0.6 & 0.2 & \\
c & 0.4 & 0.5 & 0.6 & \\
& & & & \\
(H, A) \odot(H, A) & h_{1} & h_{4} & h_{5} \\
b & & 0.0 & 0.2 & 0.0 \\
c & & 0.0 & 0.0 & 0.2
\end{array}
$$

Define a soft $L$-neighborhood system $N: X \rightarrow\left(L^{A}\right)^{S(X, A)}$ as follows

$$
\begin{gathered}
N_{h_{1}}((F, A))= \begin{cases}(1,1), & \text { if }(F, A)=(\overline{1}, A) \\
(0.5,0.4), & \text { if }(H, A) \leq(F, A), \\
(0,0), & \text { otherwise, }\end{cases} \\
N_{h_{4}}((F, A))= \begin{cases}(1,1), & \text { if }(F, A)=(\overline{1}, A) \\
(0.6,0.5), & \text { if }(H, A) \leq(F, A), \\
(0.2,0.0), & \text { if }(H, A) \odot(H, A) \leq(F, A), \\
(0,0), & \text { otherwise, }\end{cases} \\
N_{h_{5}}((F, A))= \begin{cases}(1,1), & \text { if }(F, A)=(\overline{1}, A) \\
(0.2,0.6), & \text { if }(H, A) \leq(F, A), \\
(0.0,0.2), & \text { if }(H, A) \odot(H, A) \leq(F, A), \\
(0,0), & \text { otherwise, }\end{cases}
\end{gathered}
$$

We obtain $\left(U_{H}, A\right),\left(U_{H \odot H}, A\right) \in S(X \times X, A)$ such that, for $a \in A, U_{H}(a) \in$ $L^{X \times X}$ with $U_{H}(a)(x, y)=H(a)(x) \rightarrow H(a)(y)$,

$$
U_{H}(b)=\left(\begin{array}{ccc}
1 & 1 & 0.7 \\
0.9 & 1 & 0.6 \\
1 & 1 & 1
\end{array}\right) \quad U_{H}(c)=\left(\begin{array}{ccc}
1 & 1 & 1 \\
0.9 & 1 & 1 \\
0.8 & 0.9 & 1
\end{array}\right)
$$




$$
U_{H \odot H}(b)=\left(\begin{array}{ccc}
1 & 1 & 1 \\
0.8 & 1 & 0.8 \\
1 & 1 & 1
\end{array}\right) \quad U_{H \odot H}(c)=\left(\begin{array}{ccc}
1 & 1 & 1 \\
1 & 1 & 1 \\
0.8 & 0.8 & 1
\end{array}\right)
$$

By Theorem 3.2, for $\left(U_{H}, A\right) \leq(U, A) \neq\left(1_{X \times X}, A\right)$, we have

$$
\begin{aligned}
& \mathcal{U}_{N}((U, A)) \\
& =\bigvee\left\{\bigwedge_{a \in A} \bigvee_{x \in X} N_{x}((H, A))(a) \mid\left(U_{H}, A\right) \leq(U, A)\right\} \\
& =N_{h_{1}}((H, A))(a) \vee N_{h_{4}}((H, A))(a) \vee N_{h_{5}}((H, A))(a) \\
& \vee N_{h_{1}}((H, A))(b) \vee N_{h_{4}}((H, A))(b) \vee N_{h_{5}}((H, A))(b) \\
& =0.5 \vee 0.6 \vee 0.2 \vee 0.4 \vee 0.5 \vee 0.6=0.6 .
\end{aligned}
$$

By a similar method, we obtain $\mathcal{U}_{N}: S(X \times X, A) \rightarrow L$ as follows

$$
\begin{aligned}
& \mathcal{U}_{N}((U, A)) \\
& = \begin{cases}1, & \text { if }(U, A)=\left(1_{X \times X}, A\right), \\
0.6, & \text { if }\left(U_{H}, A\right) \leq(U, A) \neq\left(1_{X \times X}, A\right), \\
0.2, & \text { if }\left(U_{H \odot H}, A\right) \leq(U, A) \ngtr\left(U_{H}, A\right), \\
0.2, & \text { if }\left(U_{H}, A\right) \odot\left(U_{H}, A\right) \leq(U, A) \ngtr\left(U_{H}, A\right), \\
0, & \text { otherwise. }\end{cases}
\end{aligned}
$$

Since $U_{H}(b) \circ U_{H}(x)=U_{H}(x)$ and $U_{H \odot H}(x) \circ U_{H \odot H}(x)=U_{H \odot H}(x)$ for $x \in$ $\{a, b\}, \mathcal{U}_{N}$ is an soft $L$-fuzzy quasi-uniformity.

\section{References}

[1] K.V. Babitha, J.J. Sunil, Soft set relations and functions, Compu. Math. Appl., 60(2010), 1840-1849, doi: 10.1016/j.camwa.2010.07.014.

[2] R. Bělohlávek, Fuzzy Relational Systems, Kluwer Academic Publishers, New York, (2002), doi: 10.1007/978-1-4615-0633-1.

[3] N. Cag̈man, S. Karatas and S. Enginoglu, Soft topology, Comput. Math. Appl., 62(1) (2011), 351-358. doi: 10.1016/j.camwa.2011.05.016.

[4] F. Feng, X. Liu, V.L. Fotea, Y.B. Jun, Soft sets and soft rough sets, Information Sciences, 181 (2011), 1125-1137, doi: 10.1016/j.ins.2010.11.004.

[5] P. Hájek, Metamathematices of Fuzzy Logic, Kluwer Academic Publishers, Dordrecht (1998), doi: 10.1007/978-94-011-5300-3.

[6] U. Höhle, S.E. Rodabaugh, Mathematics of Fuzzy Sets: Logic, Topology, and Measure Theory, The Handbooks of Fuzzy Sets Series 3, Kluwer Academic Publishers, Boston, 1999, doi: 10.1007/978-1-4615-5079-2.

[7] B. Hutton, Uniformities in fuzzy topological spaces, J. Math. Anal. Appl., 58 (1977), 74-79. doi: $10.1016 / 0022-247 \times(77) 90192-5$. 
[8] A.K. Katsaras, On fuzzy uniform spaces, J. Math. Anal. Appl., 101, 1984, 97-113. doi: 10.1016/0022-247x(84)90060-x.

[9] Y.C. Kim and J.M. Ko, Soft $L$-topologies and soft $L$-neighborhood systems, (accepted to) J. Math. Comput. Sci.

[10] Y.C. Kim and J.M. Ko, Soft $L$-fuzzy quasi-uniformities and soft $L$-fuzzy topogenous orders, (submit to) J. Intelligent and Fuzzy Systems.

[11] R. Lowen, Fuzzy uniform spaces, J. Math. Anal. Appl., 82 (1981), 370-385, doi: $10.1016 / 0022-247 x(81) 90202-x$.

[12] R. Lowen, Fuzzy neighborhood spaces, Fuzzy Sets and Systems, 7 (1982), 165-189.

[13] D. Molodtsov, Soft set theory, Comput. Math. Appl., 37(1999), 19-31.

[14] Z. Pawlak, Rough sets, Int. J. Comput. Inf. Sci., 11 (1982), 341-356.

[15] Z. Pawlak, Rough probability, Bull. Pol. Acad. Sci. Math., 32(1984), 607-615.

[16] M. Shabir and M. Naz, On soft topological spaces, Comput. Math. Appl.,61 (2011), 1786-1799, doi: 10.1016/j.camwa.2011.02.006.

[17] B. Tanay and M. B. Kandemir, Topological structure of fuzzy soft sets, Comput. Math. Appl., 61(10) (2011), 2952-2957, doi: 10.1016/j.camwa.2011.03.056.

[18] W.Z. Wu and W.X. Zhang, Neighborhood operator systems and approximations, Information Sciences, 144 (2002), 201-217.

[19] $\mathrm{Hu}$ Zhao and Sheng-Gang Li, L-fuzzifying soft topological spaces and L-fuzzifying soft interior operators, Ann. Fuzzy Math. Inform., 5(3) (2013), 493-503.

[20] Í. Zorlutuna, M. Akdag, W. K. Min and S. Atmaca, Remarks on soft topological spaces, Ann. Fuzzy Math. Inform., 3(2) (2012), 171-185. 